\title{
The backlund transformation of the generalized Riccati equation and its applications to the nonlinear KPP equation
}

\begin{abstract}
The Bäcklund transformation of the generalized Riccati equation is applied in this article to construct many new exact traveling wave solutions for the nonlinear Kolmogorov-Petrovskii-Piskunov (KPP) equation. Solutions, trigonometric and rational solutions of this equation are obtained. This transformation is straightforward and concise. It gives much more general results than the well-known results obtaining by other methods. With the aid of Maple, some graphical representations for some results are presented by choosing suitable values of parameters.
\end{abstract}

Keywords: exact traveling wave solutions, bäcklund transformation of generalized Riccati equation, kolmogorov-petrovskii-piskunov equation, soliton solutions, trigonometric solutions, rational solutions
Volume I Issue I - 2017

\section{Elsayed ME Zayed,' Khaled AE Alurrfi, ${ }^{2}$ Abdul Ghani Al-Nowehy ${ }^{3}$}

'Department of Mathematics, Faculty of Sciences, Zagazig University, Egypt

${ }^{2}$ Department of Mathematics, Faculty of Arts \& Science, Mergib University, Libya

${ }^{3}$ Department of Mathematics, Faculty of Education and Science, Taiz University, Yemen

Correspondence: Elsayed ME Zayed, Department of Mathematics, Faculty of Sciences, Zagazig University, Zagazig, Egypt,Email eme_zayed@yahoo.com

Received: June 17,2017 | Published: August 30, 2017
Mathematics subject classification: 35K99, 35P05, 35P99, 35C05.

\section{Introduction}

The investigation of exact traveling wave solutions to nonlinear PDEs plays an important role in the study of nonlinear physical phenomena. Nonlinear wave phenomena appears in various scientific and engineering fields, such as fluid mechanics, plasma physics, optical fibers, biology, solid state physics, chemical kinematics, chemical physics and geochemistry. Nonlinear wave phenomena of dispersion, dissipation, diffusion, reaction and convection are very important in nonlinear wave equations. In recent decades, many effective methods have been established to obtain exact solutions of nonlinear PDEs, such as the inverse scattering transform, ${ }^{1}$ the Hirota method, ${ }^{2}$ the truncated Painlevé expansion method, ${ }^{3}$ the Bäcklund transform method, ${ }^{1,4,5}$ the exp-function method, ${ }^{6-8}$ the simplest equation method,,${ }^{9,10}$ the Weierstrass elliptic function method, ${ }^{11}$ the Jacobi elliptic function method, ${ }^{12-14}$ the tanh-function method, ${ }^{15,16}$ the $\left(G^{\prime} / \mathrm{G}\right)$ expansion method, ${ }^{17-22}$ the modified simple equation method, ${ }^{23-26}$ the Kudryashov method, ${ }^{27-29}$ the multiple exp-function algorithm method, ${ }^{30,31}$ the transformed rational function method, ${ }^{32}$ the Frobenius decomposition technique, ${ }^{33}$ the local fractional variation iteration method, ${ }^{34}$ the local fractional series expansion method, ${ }^{35}$ the $\left(\frac{G^{\prime}}{G}, \frac{1}{G}\right)$ expansion method, ${ }^{36-40}$ the generalized Riccati equation mapping method ${ }^{41-45}$ and so on.

The objective of this article is to use the Bäcklund transformation of the generalized Riccati equation to construct new exact traveling wave solutions of the following nonlinear Kolmogorov-PetrovskiiPiskunov (KPP) equation. . $2,26,46^{2}$

$$
u_{t}-u_{x x}+\mu u^{2}+\gamma u^{2}+\delta u^{3}=0
$$

Where $\mu, \gamma, \delta$ are real constants. Equation (1.1) includes the Fisher equation, Huxley equation, Burgers-Huxley equation, ChaffeeInfanfe equation and Fitzhugh-Nagumo equation as special cases. Recently, Feng et al. ${ }^{22}$ have discussed Equation (1.1) using the $\left(G^{\prime} / \mathrm{G}\right)$-expansion method and found its exact solutions, while Zayed et al. ${ }^{26,46}$ have applied two methods via the modified simple equation method and the Riccati equation method combined with the $\left(G^{\prime} / \mathrm{G}\right)$-expansion method respectively, to Equation (1.1) and determined the exact traveling wave solutions of it.

This paper is organized as follows: In Section 2, the description of the Bäcklund transformation of the generalized Riccati equation is given. In Section 3, we use the given method described in Section 2 , to find many new exact traveling wave solutions of the nonlinear KPP equation. In Section 4, physical explanations of some results are presented. In Section 5, some conclusions are obtained.

\section{Description of the båcklund transformation of the generalized riccati equation}

Suppose that we have the following nonlinear PDE:

$$
F\left(u, u_{t}, u_{x}, u_{t t}, u_{x x}, \ldots\right)=0,
$$

Where $F$ is a polynomial in $u(x, t)$ and its partial derivatives, in which the highest order derivatives and the nonlinear terms are involved. In the following, we give the main steps of this method: ${ }^{5}$ 
Step 1: Using the wave transformation

$$
u(x, t)=u(\xi), \quad \xi=k x+\omega t,
$$

where $k$ and $\omega$ are constants, to reduce Equation (2.1) to the following ODE:

$$
P\left(u, u^{\prime}, u^{\prime \prime}, \ldots\right)=0,
$$

where $P$ is a polynomial in $u(\xi)$ and its total derivatives while ' $=d / d \xi$.

Step 2: Assume that Equation (2.3) has the formal solution

$$
u(\xi)=\sum_{i=0}^{N} a_{i} \psi(\xi)^{i}
$$

where $a_{i}$ are constants to be determined, such that $a_{N} \neq 0$, while $\psi(\xi)$ comes from the following Bäcklund transformation

$$
\psi(\xi)=\frac{-r B+A \varphi(\xi)}{A+B \varphi(\xi)}
$$

where $r, A, B$ are constants with $B \neq 0$, while $\varphi(\xi)$ satisfies the generalized Riccati equation:

$$
\varphi^{\prime}(\xi)=r+p \varphi(\xi)+q \varphi(\xi)^{2},
$$

where $p, q$ are constants, such that $q \neq 0$.

It is well-known ${ }^{41-45}$ that Equation (2.6) has many families of solutions as follows:

Family 1: When $p^{2}-4 q r>0$ and $p q \neq 0$ or $q r \neq 0$, we have

$$
\begin{aligned}
& \varphi_{1}(\xi)=-\frac{1}{2 q}\left(p+\sqrt{p^{2}-4 q r} \tanh \left(\frac{\sqrt{p^{2}-4 q r}}{2} \xi\right)\right), \\
& \varphi_{2}(\xi)=-\frac{1}{2 q}\left(p+\sqrt{p^{2}-4 q r} \operatorname{coth}\left(\frac{\sqrt{p^{2}-4 q r}}{2} \xi\right)\right) \text {, } \\
& \varphi_{3}(\xi)=-\frac{1}{2 q}\left(p+\sqrt{p^{2}-4 q r}\left(\operatorname{coth}\left(\sqrt{p^{2}-4 q r} \xi\right) \pm \operatorname{csch}\left(\sqrt{p^{2}-4 q r} \xi\right)\right)\right. \text {, } \\
& \varphi_{4}(\xi)=-\frac{1}{4 q}\left(2 p+\sqrt{p^{2}-4 q r}\left(\tanh \left(\frac{\sqrt{p^{2}-4 q r}}{4} \xi\right)+\operatorname{coth}\left(\frac{\sqrt{p^{2}-4 q r}}{4} \xi\right)\right)\right. \text {, } \\
& \varphi_{5}(\xi)=\frac{1}{2 q}\left(-p+\frac{ \pm \sqrt{\left(R^{2}+M^{2}\right)\left(p^{2}-4 q r\right)}-A \sqrt{p^{2}-4 q r} \cosh \left(\sqrt{p^{2}-4 q r} \xi\right)}{R \sinh \left(\sqrt{p^{2}-4 q r} \xi\right)+M}\right), \\
& \varphi_{6}(\xi)=\frac{1}{2 q}\left(-p-\frac{ \pm \sqrt{\left(M^{2}-R^{2}\right)\left(p^{2}-4 q r\right)}+A \sqrt{p^{2}-4 q r} \sinh \left(\sqrt{p^{2}-4 q r} \xi\right)}{R \cosh \left(\sqrt{p^{2}-4 q r} \xi\right)+M}\right) \text {, }
\end{aligned}
$$

Where $R$ and $M$ are nonzero real constants satisfying $M^{2}-R^{2}>0$

$$
\begin{gathered}
\varphi_{7}(\xi)=\frac{2 r \cosh \left(\frac{\sqrt{p^{2}-4 q r}}{2} \xi\right)}{\sqrt{p^{2}-4 q r} \sinh \left(\frac{\sqrt{p^{2}-4 q r}}{2} \xi\right)-p \cosh \left(\frac{\sqrt{p^{2}-4 q r}}{2} \xi\right)}, \\
\varphi_{8}(\xi)=\frac{-2 r \sinh \left(\frac{\sqrt{p^{2}-4 q r}}{2} \xi\right)}{2 \sinh \left(\frac{\sqrt{p^{2}-4 q r}}{2} \xi\right)-\sqrt{p^{2}-4 q r} \cosh \left(\frac{\sqrt{p^{2}-4 q r}}{2} \xi\right)}, \\
\varphi_{9}(\xi)=\frac{2 r \sinh \left(\sqrt{p^{2}-4 q r} \xi\right)}{-p \sinh \left(\sqrt{p^{2}-4 q r} \xi\right)+\sqrt{p^{2}-4 q r} \cosh \left(\sqrt{p^{2}-4 q r} \xi\right) \pm \sqrt{p^{2}-4 q r}}, \\
\varphi_{10}(\xi)=\frac{4 r \sinh \left(\frac{1}{4} \sqrt{p^{2}-4 q r} \xi\right) \cosh \left(\frac{1}{4} \sqrt{p^{2}-4 q r} \xi\right)}{-2 p \sinh \left(\frac{1}{4} \sqrt{p^{2}-4 q r} \xi\right) \cosh \left(\frac{1}{4} \sqrt{p^{2}-4 q r} \xi\right)+2 \sqrt{p^{2}-4 q r} \cosh ^{2}\left(\frac{1}{4} \sqrt{p^{2}-4 q r} \xi\right)-\sqrt{p^{2}-4 q r}} .
\end{gathered}
$$

Family 2: When $p^{2}-4 q r<0$ and $p q \neq 0$ or $q r \neq 0$, we have

$$
\begin{aligned}
& \varphi_{11}(\xi)=\frac{1}{2 q}\left(-p+\sqrt{4 q r-p^{2}} \tan \left(\frac{\sqrt{4 q r-p^{2}}}{2} \xi\right)\right. \text {, } \\
& \varphi_{12}(\xi)=-\frac{1}{2 q}\left(p+\sqrt{4 q r-p^{2}} \cot \left(\frac{\sqrt{4 q r-p^{2}}}{2} \xi\right)\right. \text {, } \\
& \varphi_{13}(\xi)=\frac{1}{2 q}\left(-p+\sqrt{4 q r-p^{2}}\left(\tan \left(\sqrt{4 q r-p^{2}} \xi\right) \pm \sec \left(\sqrt{4 q r-p^{2}} \xi\right)\right),\right. \\
& \varphi_{14}(\xi)=-\frac{1}{2 q}\left(p+\sqrt{4 q r-p^{2}}\left(\cot \left(\sqrt{4 q r-p^{2}} \xi\right) \pm \csc \left(\sqrt{4 q r-p^{2}} \xi\right)\right)\right), \\
& \varphi_{15}(\xi)=\frac{1}{4 q}\left(-2 p+\sqrt{4 q r-p^{2}}\left(\tan \left(\frac{\sqrt{4 q r-p^{2}}}{4} \xi\right)-\cot \left(\frac{\sqrt{4 q r-p^{2}}}{4} \xi\right)\right),\right. \\
& \varphi_{16}(\xi)=\frac{1}{2 q}\left(-p+\frac{ \pm \sqrt{\left(R^{2}-M^{2}\right)\left(4 q r-p^{2}\right)}-A \sqrt{4 q r-p^{2}} \cos \left(\sqrt{4 q r-p^{2}} \xi\right)}{R \sin \left(\sqrt{4 q r-p^{2}} \xi\right)+M}\right), \\
& \varphi_{17}(\xi)=\frac{1}{2 q}\left(-p-\frac{ \pm \sqrt{\left(R^{2}-M^{2}\right)\left(4 q r-p^{2}\right)}+A \sqrt{4 q r-p^{2}} \sin \left(\sqrt{4 q r-p^{2}} \xi\right)}{R \cos \left(\sqrt{4 q r-p^{2}} \xi\right)+M}\right),
\end{aligned}
$$

where $R$ and $M$ are two nonzero real constants satisfying $R^{2}-M^{2}>0$. 


$$
\begin{aligned}
& \varphi_{18}(\xi)=\frac{-2 r \cos \left(\frac{\sqrt{4 q r-p^{2}}}{2} \xi\right)}{\sqrt{4 q r-p^{2}} \sin \left(\frac{\sqrt{4 q r-p^{2}}}{2} \xi\right)+p \cos \left(\frac{\sqrt{4 q r-p^{2}}}{2} \xi\right)}, \\
& D\left[\frac{d^{l} u}{d \xi^{l}}\right]=N+l, \\
& D\left[u^{m}\left(\frac{d^{l} u}{d \xi^{l}}\right)^{s}\right]=N m+s(l+N) \text {. }
\end{aligned}
$$$$
\varphi_{19}(\xi)=\frac{2 r \sin \left(\frac{\sqrt{4 q r-p^{2}}}{2} \xi\right)}{-p \sin \left(\frac{\sqrt{4 q r-p^{2}}}{2} \xi\right)+\sqrt{4 q r-p^{2}} \cos \left(\frac{\sqrt{4 q r-p^{2}}}{2} \xi\right)},
$$$$
\varphi_{20}(\xi)=\frac{-2 r \cos \left(\sqrt{4 q r-p^{2}} \xi\right)}{\sqrt{4 q r-p^{2}} \sin \left(\sqrt{4 q r-p^{2}} \xi\right)+p \cos \left(\sqrt{4 q r-p^{2}} \xi\right) \pm \sqrt{4 q r-p^{2}}} .
$$$$
\varphi_{21}(\xi)=\frac{2 r \sin \left(\sqrt{4 q r-p^{2}} \xi\right)}{-p \sin \left(\sqrt{4 q r-p^{2}} \xi\right)+\sqrt{4 q r-p^{2}} \cos \left(\sqrt{4 q r-p^{2}} \xi\right) \pm \sqrt{4 q r-p^{2}}},
$$$$
\varphi_{22}(\xi)=\frac{4 r \sin \left(\frac{1}{4} \sqrt{4 q r-p^{2}} \xi\right) \cos \left(\frac{1}{4} \sqrt{4 q r-p^{2}} \xi\right)}{-2 p \sin \left(\frac{1}{4} \sqrt{4 q r-p^{2}} \xi\right) \cos \left(\frac{1}{4} \sqrt{4 q r-p^{2}} \xi\right)+2 \sqrt{4 q r-p^{2}} \cos ^{2}\left(\frac{1}{4} \sqrt{4 q r-p^{2}} \xi\right)-\sqrt{4 q r-p^{2}}}
$$

Family 3: When $r=0$ and $p q \neq 0$, we have

$$
\begin{aligned}
\varphi_{23}(\xi) & =\frac{-p d}{q(d+\cosh (p \xi)-\sinh (p \xi))} \\
\varphi_{24}(\xi) & =\frac{-p(\cosh (p \xi)+\sinh (p \xi))}{q(d+\cosh (p \xi)+\sinh (p \xi))},
\end{aligned}
$$

Where $d$ is an arbitrary constant.

Family 4: When $q \neq 0$ and $r=p=0$, we have

$$
\varphi_{25}(\xi)=\frac{-1}{q \xi+c_{1}}
$$

Where $c_{1}$ is an arbitrary constant.

Step 3: We determine the positive integer $N$ in (2.4) by using the homogeneous balance between the highest-order derivatives and the nonlinear terms in Equation (2.3). More precisely we define the degree of $u(\xi)$ as $D[u(\xi)]=N$ which gives rise to the degree of other expressions as follows:
Therefore, we can get the value of $N$ in

Step 4: We substitute (2.4) along with Equations (2.5) and (2.6) into Equation (2.3), collect all the terms with the same powers of $\varphi^{i}(\xi)$ and set them to zero, we obtain a system of algebraic equations, which can be solved by Maple to get the values of $a_{i}, k$ and $\omega$. Consequently, we obtain the exact traveling wave solutions of Equation (2.1).

\section{An application}

In this section, we will apply the method described in Section 2 to find the exact traveling wave solutions of the nonlinear KPP equation (1.1). To this end, we use the wave transformation (2.2) to reduce Equation (1.1) to the following ODE:

$$
\omega u^{\prime}(\xi)-k^{2} u^{\prime \prime}(\xi)+\mu u(\xi)+\gamma u^{2}(\xi)+\delta u^{3}(\xi)=0 .
$$

By balancing $u^{\prime \prime}$ with $u^{3}$ in Equation (3.1), we get $N=1$. Consequently, we have the formal solution

$$
u(\xi)=a_{0}+a_{1} \psi(\xi)
$$

where $a_{0}, a_{1}$ are constants to be determined, such that $a_{1} \neq 0$, while $\psi(\xi)$ is given by $(2.5)$.

Now, substituting (3.2) along with Equations (2.5) and (2.6) into (3.1), collecting the coefficients of $\varphi^{i}(\xi)$ and setting them to zero, we get the following system of algebraic equations:

$$
\begin{aligned}
& \varphi^{3}: \delta A^{3} a_{1}^{3}-2 A^{3} k^{2} q^{2} a_{1}+p A^{2} B k^{2} q a_{1}+\omega A^{2} B q a_{1}+3 \delta A^{2} B a_{0} a_{1}^{2}+\gamma A^{2} B a_{1}^{2}-2 r A B^{2} k^{2} q^{2} a_{1} \\
& +3 \delta A B^{2} a_{0}^{2} a_{1}+2 \gamma A B^{2} a_{0} a_{1}+\mu A B^{2} a_{1}+p r B^{3} k^{2} q a_{1}+r \omega B^{3} q a_{1}+\delta B^{3} a_{0}^{3}+\gamma B^{3} a_{0}^{2}+\mu B^{3} a_{0}=0, \\
& \varphi^{2}: 3 \delta A^{3} a_{0} a_{1}^{2}-3 q A^{3} k^{2} p a_{1}+\gamma A^{3} a_{1}^{2}+q \omega A^{3} a_{1}+A^{2} B k^{2} p^{2} a_{1}+2 q A^{2} B k^{2} r a_{1}+\omega A^{2} B p a_{1} \\
& -3 \delta A^{2} B r a_{1}^{3}+6 \delta A^{2} B a_{0}^{2} a_{1}+4 \gamma A^{2} B a_{0} a_{1}+2 \mu A^{2} B a_{1}-3 q A B^{2} k^{2} p r a_{1}-6 \delta A B^{2} r a_{0} a_{1}^{2} \\
& -2 \gamma A B^{2} r a_{1}^{2}+q \omega A B^{2} r a_{1}+3 \delta A B^{2} a_{0}^{3}+3 \gamma A B^{2} a_{0}^{2}+3 \mu A B^{2} a_{0}+B^{3} k^{2} p^{2} r a_{1} \\
& +2 q B^{3} k^{2} r^{2} a_{1}+\omega B^{3} p r a_{1}-3 \delta B^{3} r a_{0}^{2} a_{1}-2 \gamma B^{3} r a_{0} a_{1}-\mu B^{3} r a_{1}=0, \\
& \varphi: \omega A^{3} p a_{1}-2 q A^{3} k^{2} r a_{1}-A^{3} k^{2} p^{2} a_{1}+3 \delta A^{3} a_{0}^{2} a_{1}+2 \gamma A^{3} a_{0} a_{1}+\mu A^{3} a_{1}+3 A^{2} B k^{2} p r a_{1} \\
& -6 \delta A^{2} B r a_{0} a_{1}^{2}-2 \gamma A^{2} B r a_{1}^{2}+\omega A^{2} B r a_{1}+3 \delta A^{2} B a_{0}^{3}+3 \gamma A^{2} B a_{0}^{2}+3 \mu A^{2} B a_{0}-A B^{2} k^{2} p^{2} r a_{1} \\
& -2 q A B^{2} k^{2} r^{2} a_{1}+\omega A B^{2} p r a_{1}+3 \delta A B^{2} r^{2} a_{1}^{3}-6 \delta A B^{2} r a_{0}^{2} a_{1}-4 \gamma A B^{2} r a_{0} a_{1}-2 \mu A B^{2} r a_{1} \\
& +3 B^{3} k^{2} p r^{2} a_{1}+3 \delta B^{3} r^{2} a_{0} a_{1}^{2}+\gamma B^{3} r^{2} a_{1}^{2}+\omega B^{3} r^{2} a_{1}=0,
\end{aligned}
$$


$\varphi^{0}: \omega A^{3} r a_{1}-p A^{3} k^{2} r a_{1}+\delta A^{3} a_{0}^{3}+\gamma A^{3} a_{0}^{2}+\mu A^{3} a_{0}+2 A^{2} B k^{2} r^{2} a_{1}-3 \delta A^{2} B r a_{0}^{2} a_{1}-2 \gamma A^{2} B r a_{0} a_{1}$ $-\mu A^{2} B r a_{1}-p A B^{2} k^{2} r^{2} a_{1}+3 \delta A B^{2} r^{2} a_{0} a_{1}^{2}+\gamma A B^{2} r^{2} a_{1}^{2}+\omega A B^{2} r^{2} a_{1}+2 B^{3} k^{2} r^{3} a_{1}-\delta B^{3} r^{3} a_{1}^{3}=0$.

On solving the above algebraic equations with the aid of Maple or Mathematical, we have the following results:

\section{Result 1:}

$$
\begin{aligned}
& a_{0}=0, a_{1}=\frac{2 k^{2} p}{\gamma}, r=\frac{\omega^{2}}{4 k^{4}}, p=p, q=-1, \delta=\frac{\gamma^{2} k^{2}}{2 \omega^{2}}, \\
& \mu=-\frac{1}{2} k^{2} p^{2}, A=-\frac{\omega B}{2 k^{2}}, B=B, k=k, \omega=\omega .
\end{aligned}
$$

Form this result, we have $p^{2}-4 q r=p^{2}+\frac{\omega^{2}}{k^{4}}>0$.

Consequently, we have the following exact solutions:

$$
\begin{gathered}
u_{1}(\xi)=-\frac{p \omega}{\gamma}\left[\frac{\left(\omega+k^{2} p\right)+k^{2} \sqrt{p^{2}+\frac{\omega^{2}}{k^{4}}} \tanh \left(\frac{1}{2} \sqrt{p^{2}+\frac{\omega^{2}}{k^{4}} \xi}\right)}{\left.\left(-\omega+k^{2} p\right)+k^{2} \sqrt{p^{2}+\frac{\omega^{2}}{k^{4}}} \tanh \left(\frac{1}{2} \sqrt{p^{2}+\frac{\omega^{2}}{k^{4}} \xi}\right)\right],}\right. \\
u_{2}(\xi)=-\frac{p \omega}{\gamma}\left[\frac{\left(\omega+k^{2} p\right)+k^{2} \sqrt{p^{2}+\frac{\omega^{2}}{k^{4}}} \operatorname{coth}\left(\frac{1}{2} \sqrt{p^{2}+\frac{\omega^{2}}{k^{4}} \xi}\right)}{\left.\left(-\omega+k^{2} p\right)+k^{2} \sqrt{p^{2}+\frac{\omega^{2}}{k^{4}}} \operatorname{coth}\left(\frac{1}{2} \sqrt{p^{2}+\frac{\omega^{2}}{k^{4}} \xi}\right)\right]}\right] \\
\left.u_{3}(\xi)=-\frac{p \omega}{\gamma}\left[\frac{\left(\omega+k^{2} p\right)+k^{2} \sqrt{p^{2}+\frac{\omega^{2}}{k^{4}}}\left(\operatorname{coth}\left(\sqrt{p^{2}+\frac{\omega^{2}}{k^{4}}} \xi\right) \pm \operatorname{csch}\left(\sqrt{p^{2}+\frac{\omega^{2}}{k^{4}}} \xi\right)\right.}{\left(-\omega+k^{2} p\right)+k^{2} \sqrt{p^{2}+\frac{\omega^{2}}{k^{4}}}\left(\operatorname{coth}\left(\sqrt{p^{2}+\frac{\omega^{2}}{k^{4}}} \xi\right) \pm \operatorname{csch}\left(\sqrt{p^{2}+\frac{\omega^{2}}{k^{4}}} \xi\right)\right.}\right)\right],
\end{gathered}
$$$$
u_{4}(\xi)=-\frac{p \omega}{\gamma}\left[\frac{2\left(\omega+k^{2} p\right)+k^{2} \sqrt{p^{2}+\frac{\omega^{2}}{k^{4}}}\left(\tanh \left(\frac{1}{4} \sqrt{p^{2}+\frac{\omega^{2}}{k^{4}}} \xi\right)+\operatorname{coth}\left(\frac{1}{4} \sqrt{p^{2}+\frac{\omega^{2}}{k^{4}}} \xi\right)\right)}{2\left(-\omega+k^{2} p\right)+k^{2} \sqrt{p^{2}+\frac{\omega^{2}}{k^{4}}}\left(\tanh \left(\frac{1}{4 \sqrt{p^{2}+\frac{\omega^{2}}{k^{4}}}} \xi\right)+\operatorname{coth}\left(\frac{1}{4} \sqrt{p^{2}+\frac{\omega^{2}}{k^{4}}} \xi\right)\right)}\right]
$$

$$
u_{5}(\xi)=-\frac{p \omega}{\gamma}\left[\frac{\left.\left(\omega+k^{2} p\right)-k^{2}\left(\frac{ \pm \sqrt{\frac{\left(R^{2}+M^{2}\right)\left(p^{2} k^{4}+\omega^{2}\right)}{k^{4}}}-R \sqrt{p^{2}+\frac{\omega^{2}}{k^{4}}} \cosh \left(\sqrt{p^{2}+\frac{\omega^{2}}{k^{4}} \xi}\right)}{R \sinh \left(\sqrt{p^{2}+\frac{\omega^{2}}{k^{4}} \xi}\right)+M}\right)\right]}{\left(-\omega+k^{2} p\right)-k^{2}\left(\frac{ \pm \sqrt{\frac{\left(R^{2}+M^{2}\right)\left(p^{2} k^{4}+\omega^{2}\right)}{k^{4}}}-R \sqrt{p^{2}+\frac{\omega^{2}}{k^{4}}} \cosh \left(\sqrt{p^{2}+\frac{\omega^{2}}{k^{4}}}\right)}{R \sinh \left(\sqrt{p^{2}+\frac{\omega^{2}}{k^{4}} \xi}\right)+M}\right)}\right]
$$

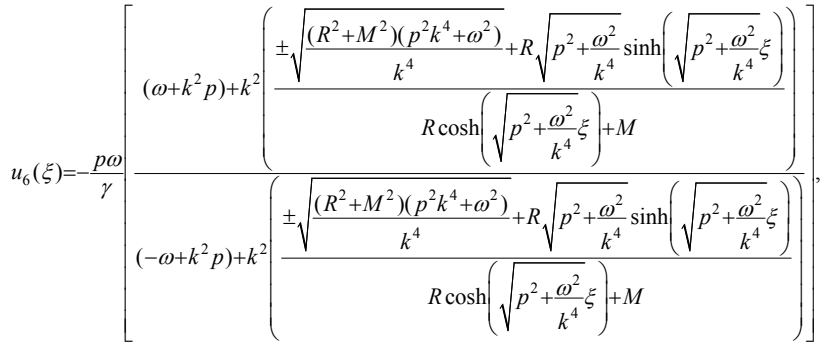

$u_{7}(\xi)=-\frac{p \omega}{\gamma}\left[\frac{\sqrt{p^{2} k^{4}+\omega^{2}} \sinh \left(\frac{1}{2} \sqrt{p^{2}+\frac{\omega^{2}}{k^{4}}} \xi\right)+\left(\omega-p k^{2}\right) \cosh \left(\frac{1}{2} \sqrt{p^{2}+\frac{\omega^{2}}{k^{4}}} \xi\right)}{-\sqrt{p^{2} k^{4}+\omega^{2}} \sinh \left(\frac{1}{2} \sqrt{p^{2}+\frac{\omega^{2}}{k^{4}}} \xi\right)+\left(\omega+p k^{2}\right) \cosh \left(\frac{1}{2} \sqrt{p^{2}+\frac{\omega^{2}}{k^{4}}} \xi\right)}\right]$, $u_{8}(\xi)=-\frac{p \omega}{\gamma}\left[\frac{\left(\omega-p k^{2}\right) \sinh \left(\frac{1}{2} \sqrt{p^{2}+\frac{\omega^{2}}{k^{4}}} \xi\right)+\sqrt{p^{2} k^{4}+\omega^{2}} \cosh \left(\frac{1}{2} \sqrt{p^{2}+\frac{\omega^{2}}{k^{4}}} \xi\right)}{\left(\omega+p k^{2}\right) \sinh \left(\frac{1}{2} \sqrt{p^{2}+\frac{\omega^{2}}{k^{4}}} \xi\right)-\sqrt{p^{2} k^{4}+\omega^{2}} \cosh \left(\frac{1}{2} \sqrt{p^{2}+\frac{\omega^{2}}{k^{4}}} \xi\right)}\right]$,

$$
u_{9}(\xi)=-\frac{p \omega}{\gamma}\left[\frac{\left(\omega-p k^{2}\right) \sinh \left(\sqrt{p^{2}+\frac{\omega^{2}}{k^{4}}} \xi\right)+\sqrt{p^{2} k^{4}+\omega^{2}}\left(\cosh \left(\sqrt{p^{2}+\frac{\omega^{2}}{k^{4}}} \xi\right) \pm 1\right)}{\left(\omega+p k^{2}\right) \sinh \left(\sqrt{p^{2}+\frac{\omega^{2}}{k^{4}}} \xi\right)-\sqrt{p^{2} k^{4}+\omega^{2}}\left(\cosh \left(\sqrt{p^{2}+\frac{\omega^{2}}{k^{4}}} \xi\right) \pm 1\right.}\right),
$$$$
u_{10}(\xi)=-\frac{p \omega}{\gamma}\left[\frac{2\left(\omega-p k^{2}\right) \sinh \left(\frac{1}{4} \sqrt{p^{2}+\frac{\omega^{2}}{k^{4}}} \xi \cosh \left(\frac{1}{4} \sqrt{p^{2}+\frac{\omega^{2}}{k^{4}}} \xi\right)+\sqrt{p^{2} k^{4}+\omega^{2}}\left(2 \cosh ^{2}\left(\frac{1}{4} \sqrt{p^{2}+\frac{\omega^{2}}{k^{4}}} \xi\right)-1\right)\right.}{2\left(\omega+p k^{2}\right) \sinh \left(\frac { 1 } { 4 } \sqrt { p ^ { 2 } + \frac { \omega ^ { 2 } } { k ^ { 4 } } } \xi \operatorname { c o s h } \left(\frac{1}{4} \sqrt{p^{2}+\frac{\omega^{2}}{k^{4}}} \xi-\sqrt{p^{2} k^{4}+\omega^{2}}\left(2 \cosh ^{2}\left(\frac{1}{4} \sqrt{p^{2}+\frac{\omega^{2}}{k^{4}}} \xi\right)-1\right)\right.\right.}\right]
$$

where $\xi=k x+\omega t$.

\section{Result 2:}

$$
\begin{aligned}
& a_{0}=0, a_{1}=\frac{\left(-\omega+3 k^{2} p\right)(A q-B p)}{\gamma A}, r=0, p=p, q=q, \delta=\frac{2 \gamma^{2} k^{2}}{\left(-\omega+3 k^{2} p\right)^{2}}, \\
& A=A, B=B, \mu=p\left(-\omega+k^{2} p\right), k=k, \omega=\omega .
\end{aligned}
$$

Since $r=0$ and $p q \neq 0$, then we have the following exact solutions:

$$
\begin{gathered}
u_{11}(\xi)=\frac{\left(-\omega+3 k^{2} p\right)(A q-B p)}{\gamma}\left[\frac{-p d}{(A q-B p) d+A q(\cosh (p \xi)-\sinh (p \xi))}\right], \\
u_{12}(\xi)=\frac{\left(-\omega+3 k^{2} p\right)(A q-B p)}{\gamma}\left[\frac{-p(\cosh (p \xi)+\sinh (p \xi))}{A q d+(A q-B p)(\cosh (p \xi)+\sinh (p \xi))}\right],
\end{gathered}
$$

where $\xi=k x+\omega t$. 


\section{Result 3:}

$$
\begin{aligned}
& a_{0}=0, a_{1}= \pm \frac{k(A q-B p) \sqrt{\frac{2}{\delta}}}{A}, r=0, p=p, q=q, A=A, B=B, \\
& \mu=k p\left(\sqrt{\frac{2 \gamma^{2}}{\delta}}-2 k p\right), k=k, \omega=-k\left(\sqrt{\frac{2 \gamma^{2}}{\delta}}-3 k p\right), \quad \delta>0 .
\end{aligned}
$$

Since $r=0$ and $p q \neq 0$, then we have the following exact solutions: $u_{13}(\xi)= \pm k(A q-B p) \sqrt{\frac{2}{\delta}}\left[\frac{-p d}{(A q-B p) d+A q(\cosh (p \xi)-\sinh (p \xi))}\right]$, $u_{14}(\xi)= \pm k(A q-B p) \sqrt{\frac{2}{\delta}}\left[\frac{-p(\cosh (p \xi)+\sinh (p \xi))}{A q d+(A q-B p)(\cosh (p \xi)+\sinh (p \xi))}\right]$ where $\quad \xi=k x-k\left(\sqrt{\frac{2 \gamma^{2}}{\delta}}-3 k p\right) t$.

\section{Result 4:}

$$
\begin{aligned}
& a_{0}=-\frac{A \gamma\left(-A B p+q A^{2}+r B^{2}\right)}{\delta(-p B+2 A q)\left(A^{2}+r B^{2}\right)}, a_{1}=\frac{B \gamma\left(-A B p+q A^{2}+r B^{2}\right)}{\delta(-p B+2 A q)\left(A^{2}+r B^{2}\right)}, r=r, \\
& p=p, q=q, A=A, B=B, \mu=\frac{q \gamma^{2}\left(-A B p+q A^{2}+r B^{2}\right)}{\delta(-p B+2 A q)^{2}}, \\
& \text { (3) } \frac{2 B \sqrt{\frac{\gamma^{2}}{8 \delta}}}{-p B+2 A q}, \omega=\frac{B \gamma^{2}}{2 \delta(-p B+2 A q)}, \quad \delta>0 .
\end{aligned}
$$

In this case, we deduce that Equation (1.1) has many types of the exact traveling wave solutions as follows:

Type 1: When $p^{2}-4 q r>0$ and $p q \neq 0$ or $q r \neq 0$, we have

$$
\begin{aligned}
& u_{15}(\xi)=-\frac{\gamma\left(-A B p+q A^{2}+r B^{2}\right)}{\delta(-p B+2 A q)\left(A^{2}+r B^{2}\right)}\left[A+\frac{B\left(2 r q B+p A+A \sqrt{p^{2}-4 r q} \tanh \left(\frac{1}{2} \sqrt{p^{2}-4 r q} \xi\right)\right)}{2 A q-p B-B \sqrt{p^{2}-4 r q} \tanh \left(\frac{1}{2} \sqrt{p^{2}-4 r q} \xi\right)},\right. \\
& u_{16}(\xi)=-\frac{\gamma\left(-A B p+q A^{2}+r B^{2}\right)}{\delta(-p B+2 A q)\left(A^{2}+r B^{2}\right)}\left[A+\frac{B\left(2 r q B+p A+A \sqrt{p^{2}-4 r q} \operatorname{coth}\left(\frac{1}{2} \sqrt{p^{2}-4 r q} \xi\right)\right)}{2 A q-p B-B \sqrt{p^{2}-4 r q} \operatorname{coth}\left(\frac{1}{2} \sqrt{p^{2}-4 r q} \xi\right)}\right]
\end{aligned}
$$

$$
\begin{gathered}
u_{17}(\xi)=-\frac{\gamma\left(-A B p+q A^{2}+r B^{2}\right)}{\delta(-p B+2 A q)\left(A^{2}+r B^{2}\right)}\left[A+\frac{B\left(2 r q B+p A+A \sqrt{p^{2}-4 r q}\left(\operatorname{coth}\left(\sqrt{p^{2}-4 r q}\right) \pm \operatorname{csch}\left(\sqrt{p^{2}-4 r q} \xi\right)\right)\right)}{2 A q-p B-B \sqrt{p^{2}-4 r q}\left(\operatorname{coth}\left(\sqrt{p^{2}-4 r q} \xi\right) \pm \operatorname{csch}\left(\sqrt{p^{2}-4 r q} \xi\right)\right)},\right. \\
u_{18}(\xi)=-\frac{\gamma\left(-A B p+q A^{2}+r B^{2}\right)}{\delta(-p B+2 A q)\left(A^{2}+r B^{2}\right)}\left[A+\frac{B\left(4 r q B+2 p A+A \sqrt{p^{2}-4 r q}\left(\operatorname{coth}\left(\frac{1}{4} \sqrt{p^{2}-4 r q} \xi\right)+\tanh \left(\frac{1}{4} \sqrt{p^{2}-4 r q} \xi\right)\right)\right)}{4 A q-2 p B-B \sqrt{p^{2}-4 r q}\left(\operatorname{coth}\left(\frac{1}{4} \sqrt{p^{2}-4 r q} \xi\right)+\tanh \left(\frac{1}{4} \sqrt{p^{2}-4 r q \xi}\right)\right)},\right.
\end{gathered}
$$$$
u_{19}(\xi)=-\frac{\gamma\left(-A B p+q A^{2}+r B^{2}\right)}{\delta(-p B+2 A q)\left(A^{2}+r B^{2}\right)}\left[A+\frac{B\left(2 r q B+p A-\frac{A \sqrt{p^{2}-4 r q}\left(-R \cosh \left(\sqrt{p^{2}-4 r q} \xi\right) \pm \sqrt{R^{2}+M^{2}}\right)}{R \sinh \left(\sqrt{p^{2}-4 r q}\right)+M}\right)}{2 A q-p B+\frac{B \sqrt{p^{2}-4 r q}\left(-R \cosh \left(\sqrt{p^{2}-4 r q} \xi\right) \pm \sqrt{R^{2}+M^{2}}\right)}{R \sinh \left(\sqrt{p^{2}-4 r q} \xi\right)+M}}\right],
$$$$
u_{20}(\xi)=-\frac{\gamma\left(-A B p+q A^{2}+r B^{2}\right)}{\delta(-p B+2 A q)\left(A^{2}+r B^{2}\right)}\left[A+\frac{B\left(2 r q B+p A+\frac{A \sqrt{p^{2}-4 r q}\left(R \sinh \left(\sqrt{p^{2}-4 r q} \xi\right) \pm \sqrt{R^{2}+M^{2}}\right)}{R \cosh \left(\sqrt{p^{2}-4 r q} \xi\right)+M}\right)}{2 A q-p B-\frac{B \sqrt{p^{2}-4 r q}\left(R \sinh \left(\sqrt{p^{2}-4 r q} \xi\right) \pm \sqrt{R^{2}+M^{2}}\right)}{R \cosh \left(\sqrt{p^{2}-4 r q} \xi\right)+M}}\right]
$$$$
u_{21}(\xi)=-\frac{\eta\left(-A B p+q A^{2}+r B^{2}\right)}{\delta(-p B+2 A q)\left(A^{2}+r B^{2}\right)}\left[A+\frac{B\left(r(p B+2 A) \cosh \left(\frac{1}{2} \sqrt{p^{2}-4 r q} \xi\right)-B r \sqrt{p^{2}-4 r q} \sinh \left(\frac{1}{2} \sqrt{p^{2}-4 r q}\right)\right)}{(p A-2 r B) \cosh \left(\frac{1}{2} \sqrt{p^{2}-4 r q} \xi\right)-A \sqrt{p^{2}-4 r q} \sinh \left(\frac{1}{2} \sqrt{p^{2}-4 r q}\right)}\right]
$$

$$
u_{22}(\xi)=-\frac{\gamma\left(-A B p+q A^{2}+r B^{2}\right)}{\delta(-p B+2 A q)\left(A^{2}+r B^{2}\right)}\left[\frac{B\left(r(p B+2 A) \sinh \left(\frac{1}{2} \sqrt{p^{2}-4 r q} \xi\right)-B r \sqrt{p^{2}-4 r q} \cosh \left(\frac{1}{2} \sqrt{p^{2}-4 r q} \xi\right)\right)}{(p A-2 r B) \sinh \left(\frac{1}{2} \sqrt{p^{2}-4 r q \xi}\right)-A \sqrt{p^{2}-4 r q} \cosh \left(\frac{1}{2} \sqrt{p^{2}-4 r q} \xi\right)},\right.
$$$$
u_{23}(\xi)=-\frac{\gamma\left(-A B p+q A^{2}+r B^{2}\right)}{\delta(-p B+2 A q)\left(A^{2}+r B^{2}\right)} A+\frac{\left.B\left(r(p B+2 A) \sinh \left(\sqrt{p^{2}-4 r q} \xi\right)-B r \sqrt{p^{2}-4 r q}\left(\cosh \left(\sqrt{p^{2}-4 r q \xi}\right) \pm 1\right)\right)\right]}{(p A-2 r B) \sinh \left(\sqrt{p^{2}-4 r q} \xi\right)-A \sqrt{p^{2}-4 r q}\left(\cosh \left(\sqrt{p^{2}-4 r q \xi}\right) \pm 1\right)} \text {, }
$$

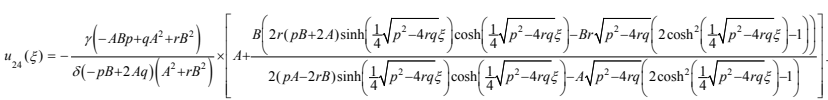

Type 2: When $p^{2}-4 q r<0$ and $p q \neq 0$ or $q r \neq 0$, we have

$$
\begin{aligned}
& u_{25}(\xi)=-\frac{\gamma\left(-A B p+q A^{2}+r B^{2}\right)}{\delta(-p B+2 A q)\left(A^{2}+r B^{2}\right)}\left[A+\frac{B\left(2 r q B+p A-A \sqrt{4 r q-p^{2}} \tan \left(\frac{1}{2} \sqrt{4 r q-p^{2}} \xi\right)\right)}{2 A q-p B+B \sqrt{4 r q-p^{2}} \tan \left(\frac{1}{2} \sqrt{4 r q-p^{2}} \xi\right)}\right], \\
& u_{26}(\xi)=-\frac{\gamma\left(-A B p+q A^{2}+r B^{2}\right)}{\delta(-p B+2 A q)\left(A^{2}+r B^{2}\right)}\left[A+\frac{B\left(2 r q B+p A+A \sqrt{4 r q-p^{2}} \cot \left(\frac{1}{2} \sqrt{4 r q-p^{2}} \xi\right)\right)}{2 A q-p B-B \sqrt{4 r q-p^{2}} \cot \left(\frac{1}{2} \sqrt{4 r q-p^{2}} \xi\right)}\right] \\
& u_{27}(\xi)=-\frac{\eta\left(-A B p+q A^{2}+r B^{2}\right)}{\delta(-p B+2 A q)\left(A^{2}+r B^{2}\right)}\left[A+\frac{B\left(2 r q B+p A-A \sqrt{4 r q-p^{2}}\left(\tan \left(\sqrt{4 r q-p^{2}} \xi\right) \pm \sec \left(\sqrt{4 r q-p^{2}} \xi\right)\right)\right)}{2 A q-p B+B \sqrt{4 r q-p^{2}}\left(\tan \left(\sqrt{4 r q-p^{2}} \xi\right) \pm \sec \left(\sqrt{4 r q-p^{2}} \xi\right)\right)}\right] \\
& u_{28}(\xi)=-\frac{\eta\left(-A B p+q A^{2}+r B^{2}\right)}{\delta(-p B+2 A q)\left(A^{2}+r B^{2}\right)}\left[A+\frac{B\left(2 r q B+p A+A \sqrt{4 r q-p^{2}}\left(\cot \left(\sqrt{4 r q-p^{2}} \xi\right) \pm \csc \left(\sqrt{4 r q-p^{2}} \xi\right)\right)\right)}{2 A q-p B-B \sqrt{4 r q-p^{2}}\left(\cot \left(\sqrt{4 r q-p^{2}} \xi\right) \pm \csc \left(\sqrt{4 r q-p^{2}} \xi\right)\right)}\right]
\end{aligned}
$$




$$
\begin{aligned}
& u_{29}(\xi)=-\frac{\gamma\left(-A B p+q A^{2}+r B^{2}\right)}{\delta(-p B+2 A q)\left(A^{2}+r B^{2}\right)}\left[A+\frac{B\left(2(2 r q B+p A)+A \sqrt{4 r q-p^{2}}\left(\cot \left(\frac{1}{4} \sqrt{4 r q-p^{2}} \xi\right)-\tan \left(\frac{1}{4} \sqrt{4 r q-p^{2}} \xi\right)\right)\right)}{2(2 A q-p B)-B \sqrt{4 r q-p^{2}}\left(\cot \left(\frac{1}{4} \sqrt{4 r q-p^{2}} \xi\right)-\tan \left(\frac{1}{4} \sqrt{4 r q-p^{2}} \xi\right)\right)}\right], \\
& u_{30}(\xi)=-\frac{\gamma\left(-A B p+q A^{2}+r B^{2}\right)}{\delta(-p B+2 A q)\left(A^{2}+r B^{2}\right)} \times\left[A+\frac{B\left((2 r q B+p A)\left(M+R \sin \left(\sqrt{4 r q-p^{2}} \xi\right)\right)-A \sqrt{4 r q-p^{2}}\left(-R \cos \left(\sqrt{4 r q-p^{2}} \xi\right) \pm \sqrt{R^{2}-M^{2}}\right)\right)}{(2 A q-p B)\left(M+R \sin \left(\sqrt{4 r q-p^{2}} \xi\right)\right)+B \sqrt{4 r q-p^{2}}\left(-R \cos \left(\sqrt{4 r q-p^{2}} \xi\right) \pm \sqrt{R^{2}-M^{2}}\right)},\right. \\
& u_{31}(\xi)=-\frac{\gamma\left(-A B p+q A^{2}+r B^{2}\right)}{\delta(-p B+2 A q)\left(A^{2}+r B^{2}\right)} \times\left[A+\frac{\left.B\left((2 r q B+p A)\left(M+R \sin \left(\sqrt{4 r q-p^{2}} \xi\right)\right)+A \sqrt{4 r q-p^{2}}\left(R \cos \left(\sqrt{4 r q-p^{2}} \xi\right) \pm \sqrt{R^{2}-M^{2}}\right)\right)\right]}{(2 A q-p B)\left(M+R \sin \left(\sqrt{4 r q-p^{2}} \xi\right)\right)-B \sqrt{4 r q-p^{2}}\left(R \cos \left(\sqrt{4 r q-p^{2}} \xi\right) \pm \sqrt{R^{2}-M^{2}}\right)}\right] \\
& u_{32}(\xi)=-\frac{\gamma\left(-A B p+q A^{2}+r B^{2}\right)}{\delta(-p B+2 A q)\left(A^{2}+r B^{2}\right)}\left[A+\frac{B\left(r(2 A+p B) \cos \left(\frac{1}{2} \sqrt{4 r q-p^{2}} \xi\right)+B r \sqrt{4 r q-p^{2}} \sin \left(\frac{1}{2} \sqrt{4 r q-p^{2}} \xi\right)\right)}{(A p-2 r B) \cos \left(\frac{1}{2} \sqrt{4 r q-p^{2}} \xi\right)+A \sqrt{4 r q-p^{2}} \sin \left(\frac{1}{2} \sqrt{4 r q-p^{2}} \xi\right)}\right] \\
& u_{33}(\xi)=-\frac{\gamma\left(-A B p+q A^{2}+r B^{2}\right)}{\delta(-p B+2 A q)\left(A^{2}+r B^{2}\right)} \mid A+\frac{B\left(r ( 2 A + p B ) \operatorname { s i n } \left(\frac{1}{2} \sqrt{4 r q-p^{2}}\right.\right.}{(A p-2 r B) \sin \left(\frac{1}{2} \sqrt{4 r q-p^{2}}\right.} \\
& u_{34}(\xi)=-\frac{\gamma\left(-A B p+q A^{2}+r B^{2}\right)}{\delta(-p B+2 A q)\left(A^{2}+r B^{2}\right)}\left[A+\frac{B\left(r(2 A+p B) \cos \left(\sqrt{4 r q-p^{2}} \xi\right)+B r \sqrt{4 r q-p^{2}}\left(\sin \left(\sqrt{4 r q-p^{2}} \xi\right) \pm 1\right)\right)}{(A p-2 r B) \cos \left(\sqrt{4 r q-p^{2}} \xi\right)+A \sqrt{4 r q-p^{2}}\left(\sin \left(\sqrt{4 r q-p^{2}} \xi\right) \pm 1\right)}\right] \\
& u_{35}(\xi)=-\frac{\gamma\left(-A B p+q A^{2}+r B^{2}\right)}{\delta(-p B+2 A q)\left(A^{2}+r B^{2}\right)}\left[A+\frac{B\left(r(2 A+p B) \sin \left(\sqrt{4 r q-p^{2}} \xi\right)-B r \sqrt{4 r q-p^{2}}\left(\cos \left(\sqrt{4 r q-p^{2}} \xi\right) \pm 1\right)\right)}{(A p-2 r B) \sin \left(\sqrt{4 r q-p^{2}} \xi\right)-A \sqrt{4 r q-p^{2}}\left(\cos \left(\sqrt{4 r q-p^{2}} \xi\right) \pm 1\right)},\right. \\
& u_{36}(\xi)=-\frac{\gamma\left(-A B p+q A^{2}+r B^{2}\right)}{\delta(-p B+2 A q)\left(A^{2}+r B^{2}\right)} \times\left[\frac{B\left(2 r(2 A+p B) \sin \left(\frac{1}{4} \sqrt{4 r q-p^{2}} \xi\right) \cos \left(\frac{1}{4} \sqrt{4 r q-p^{2}} \xi\right)-B r \sqrt{4 r q-p^{2}}\left(2 \cos ^{2}\left(\frac{1}{4} \sqrt{4 r q-p^{2}} \xi\right)-1\right)\right)}{2(A p-2 r B) \sin \left(\frac{1}{4} \sqrt{4 r q-p^{2}} \xi\right)\left(\frac{1}{4} \sqrt{4 r q-p^{2}} \xi\right)-A \sqrt{4 r q-p^{2}}\left(2 \cos ^{2}\left(\frac{1}{4} \sqrt{4 r q-p^{2}} \xi\right)-1\right)}\right] .
\end{aligned}
$$

Type 3: When $r=0$ and $p q \neq 0$, we have

$$
\begin{aligned}
& u_{37}(\xi)=-\frac{\gamma(-B p+q A)}{\delta(-p B+2 A q)}\left[1+\frac{B p d}{(A q-B p) d+A q(\cosh (p \xi)-\sinh (p \xi))}\right] \\
& u_{38}(\xi)=-\frac{\gamma(-B p+q A)}{\delta(-p B+2 A q)}\left[1+\frac{B p(\cosh (p \xi)+\sinh (p \xi))}{A q d+(A q-B p)(\cosh (p \xi)+\sinh (p \xi))}\right], \\
& \text { where } \xi=\left(\frac{2 B}{-p B+2 A q} \sqrt{\frac{\gamma^{2}}{8 \delta}}\right) x+\left(\frac{B \gamma^{2}}{2 \delta(-p B+2 A q)}\right) t .
\end{aligned}
$$

Type 4: When $r=p=0$ and $q \neq 0$, we have

$$
\begin{aligned}
& u_{39}(\xi)=-\frac{\gamma}{2 \delta}\left[1+\frac{B}{A q \xi+A c_{1}-B}\right] \\
& \text { where } \xi=\frac{B}{A q} \sqrt{\frac{\gamma^{2}}{8 \delta} x+\frac{B \gamma^{2}}{4 \delta A q} t} .
\end{aligned}
$$

\section{Physical explanations of our obtained solutions}

The obtained exact traveling wave solutions for the nonlinear
KPP equation (1.1) are hyperbolic, trigonometric and rational. In this section, we have presented some graphs of the exact solutions $u_{1}(x, t), u_{7}(x, t), u_{11}(x, t), u_{14}(x, t), u_{16}(x, t), u_{19}(x, t), u_{25}(x, t)$ and $u_{31}(x, t)$ constructed by taking suitable values of involved unknown parameters to visualize the mechanism of the original equation (1.1). These solutions are kink, singular kink-shaped soliton solution, hyperbolic solutions and trigonometric solutions. For more convenience the graphical representations of these solutions are shown in the following figures 1-8.

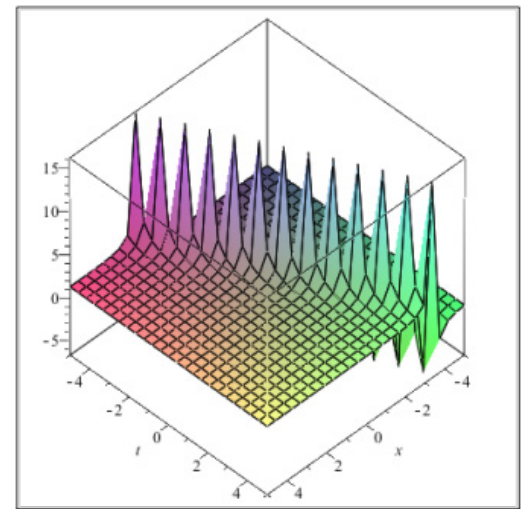

Figure I Plot of the solution $u_{1}(x, t)$ when $k=2, p=\omega=1, \gamma=-1$.

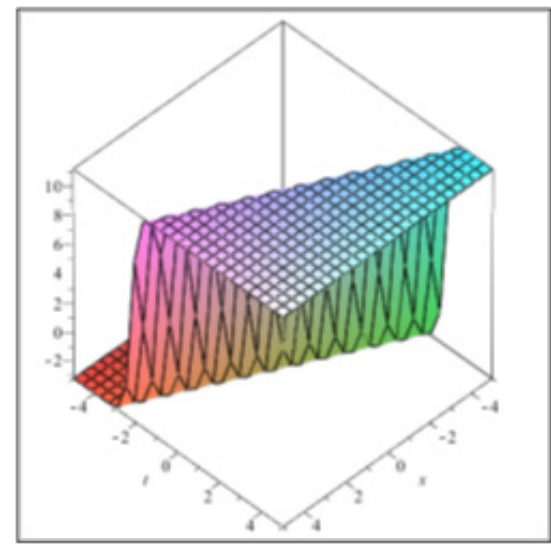

Figure 2 Plot of the solution $u_{7}(x, t)$ when $k=1, p=3, \omega=2, \gamma=-1$.

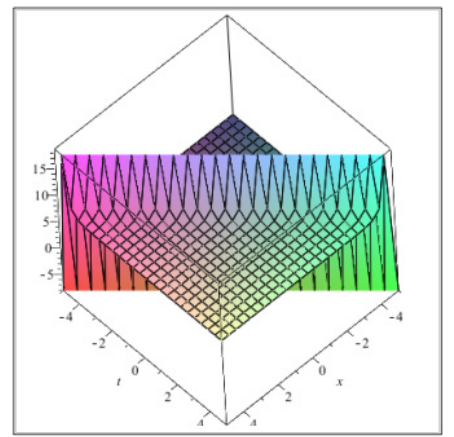

Figure 3 Plot of the solution $u_{11}(x, t)$ when $k=1, p=3, q=4, \omega=1$, $\gamma=-3, d=1, B=2, A=1$. 


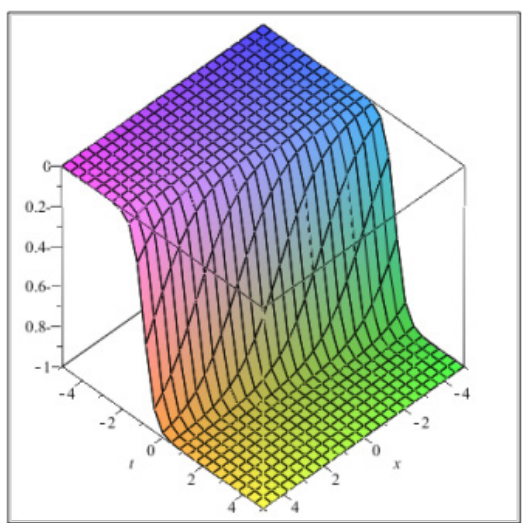

Figure 4 Plot of the solution $u_{14}(x, t)$ when $k=1, p=1, \delta=2, q=3$, $\gamma=-1, d=3, B=1, A=2$.

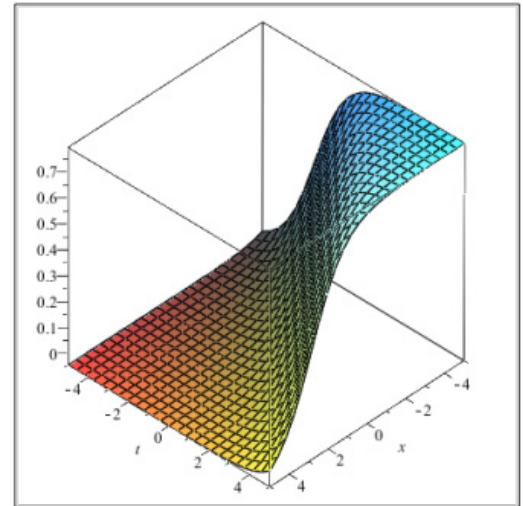

Figure 5 Plot of the solution $u_{16}(x, t)$ when $p=3, \delta=4, q=1$, $\gamma=-3, r=1, B=-2, A=-1$.

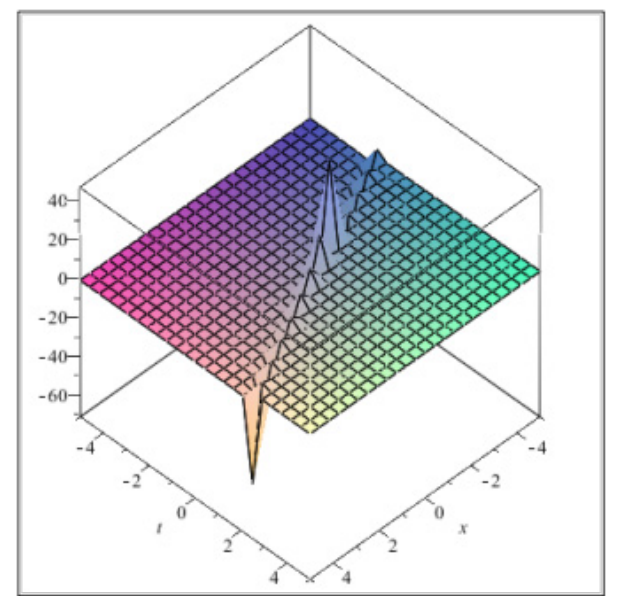

Figure 6 Plot of the solution $u_{19}(x, t)$ when

$p=5, \delta=1, q=1, \gamma=-3, r=1, B=2, A=2, R=2, \mathrm{M}=2$.

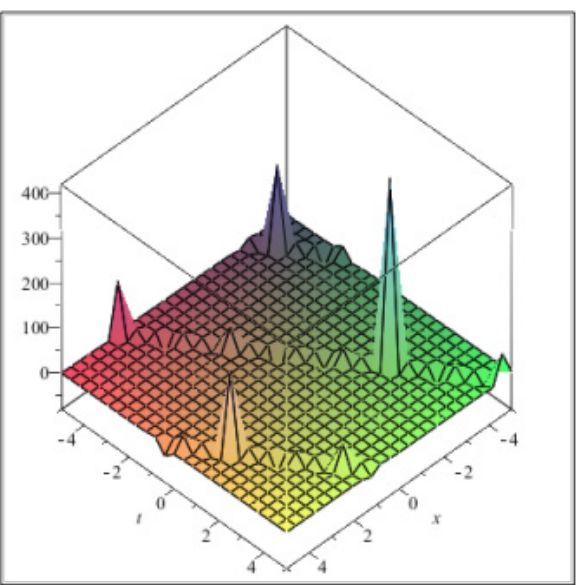

Figure 7 Plot of the solution $u_{25}(x, t)$ when $p=1, \delta=1, q=5$, $\gamma=1, r=4, B=3, A=2$.

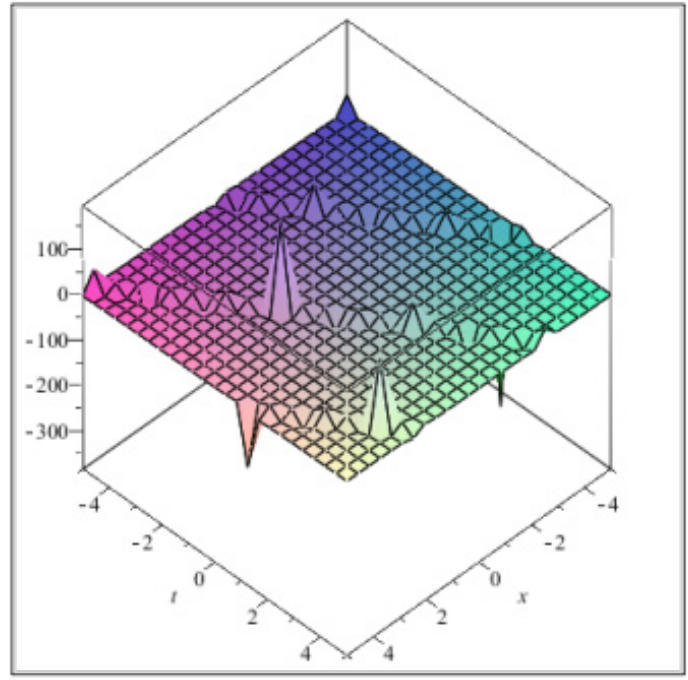

Figure 8 Plot of the solution $u_{31}(x, t)$ when $p=1, \delta=1, q=2$, $\gamma=1, r=4, B=2, A=2, R=3, M=2$.

\section{Conclusion}

In this article, we have employed the Bäcklund transformation of the generalized Riccati equation to obtain many new exact traveling wave solutions of the nonlinear Kolmogorov-Petrovskii-Piskunov (KPP) equation (1.1). On comparing our results in this paper with the well-known results obtained in ${ }^{22,26,46}$ we deduce that our results in this article are new and are not published elsewhere. The Bäcklund transformation of the generalized Riccati equation obtained in this article is more effective and gives more exact solutions than the generalized Riccati equation mapping method obtained in. ${ }^{41-45}$ Further, all solutions obtained in this article have been checked with the Maple by putting them back into the original equations. Finally, the proposed method in this article can be applied to many other nonlinear PDEs in mathematical physics, which will be done in forthcoming papers. 


\section{Acknowledgments}

None.

\section{Conflicts of interest}

The author declares there is no conflict of interest.

\section{References}

1. MJ Ablowitz, PA Clarkson. Solitons, Nonlinear Evolution Equation and Inverse Scattering. Cambridge University press, USA. 1991.

2. R Hirota. Exact solutions of $\mathrm{KdV}$ equation for multiple collisions of solitons. Physical Review Letters. 1971;27(18):1192-1194.

3. NA Kudryashov. On types of nonlinear non-integrable equations with exact solutions. Physics Letters A. 1991;155:269-275.

4. MR Miura. Bäcklund Transformation. Berlin, Springer, Germany. 1978.

5. B Lu. Bäcklund transformation of fractional Riccati equation and its applications to nonlinear fractional partial differential equations. Physics Letters A. 2012;376(28):2045-2048.

6. SA EL Wakil, MA Madkour, et al. Application of exp-function method for nonlinear evolution equations with variable coefficients. Physics Letters A 2012;369(1):62-69.

7. YP Wang. Solving the $(3+1)$-dimensional potential-YTSF equation with Exp-function method. Journal of Physics: Conference Series. 2008. p. 96.

8. K Khan, MA Akbar. Traveling wave solutions of the $(2+1)$-dimensional Zoomeron equation and the Burgers equations via the MSE method and the Exp-function method. Ain Shams Engineering Journal. 2014;5:247-256.

9. NA Kudryashov, NB Loguinova. Extended simplest equation method for nonlinear differential equations. Applied Mathematics and Computation. 2008;205:396-402.

10. YM Zhao. New exact solutions for a higher-order wave equation of $\mathrm{KdV}$ type using the multiple simplest equation method. Journal of Applied Mathematics. 2014.

11. NA Kudryashov. Exact solutions of generalized Kuramoto-Sivashinsky equation. Physics Letters A. 1990;147(5-6):287-291.

12. S Liu, Z Fu, S Liu, et al. Jacobi elliptic function expansion method and periodic wave solutions of nonlinear wave equations. Physics Letters A. 2001;289:69-74.

13. D Lu, Q Shi. New Jacobi elliptic functions solutions for the combined $\mathrm{KdV}-\mathrm{mKdV}$ equation. International Journal of Nonlinear Science. 2010;10(3):320-325.

14. EME Zayed, YA Amer, RM A Shohib. The Jacobi elliptic function expansion method and its applications for solving the higher order dispersive nonlinear Schrö dinger equation. Scientific Journal of Mathematics Research. 2010;4:53-72.

15. EJ Parkes, BR Duffy. An automated tanh-function method for finding solitary wave solutions to nonlinear evolution equations. Computer Physics Communications. 2005;698:288-300.

16. DL Sekulic, MV Sataric, MB Zivanov. Symbolic computation of some new nonlinear partial differential equations of nanobiosciences using modified extended tanh-function method. Applied Mathematics and Computation. 2010;218:3499-3506.

17. M Wang, X Li, J Zhang. The $\left(\mathrm{G}^{\prime} / \mathrm{G}\right)$ expansion method and travelling wave solutions of nonlinear evolution equations in mathematical physics. Physics Letters A. 2008;372(4):417-423.

18. EME Zayed. The $\left(\mathrm{G}^{\prime} / \mathrm{G}\right)$ expansion method and its applications to some nonlinear evolution equations in the mathematical physics. Journal of Applied Mathematics and Computing. 2009;30(1):89-103.
19. EME Zayed, KA Gepreel. The $\left(\mathrm{G}^{\prime} / \mathrm{G}\right)$ expansion method for finding traveling wave solutions of nonlinear partial differential equations in mathematical physics. Journal of Mathematical Physics. 2009.

20. ZL Li. Constructing of new exact solutions to the GKdV-mKdV equation with any-order nonlinear terms by $\left(\mathrm{G}^{\prime} / \mathrm{G}\right)$-expansion method. Applied Mathematics and Computation. 2010;217:1398-1403.

21. B Ayhan, A Bekir. The $\left(\mathrm{G}^{\prime} / \mathrm{G}\right)$ expansion method for the nonlinear lattice equations. Communications in Nonlinear Science and Numerical Simulation. 2012;17:3490-3498.

22. J Feng, W Li, Q Wan. Using $\left(\mathrm{G}^{\prime} / \mathrm{G}\right)$ expansion method to seek the traveling wave solution of Kolmogorov-Petrovskii-Piskunov equation. Applied Mathematics and Computation. 2011;217:5860-5865.

23. AJM Jawad, MD Petkovic, A Biswas. Modified simple equation method for nonlinear evolution equations. Applied Mathematics and Computation. 2010;217:869-877.

24. EME Zayed. A note on the modified simple equation method applied to Sharma-Tasso-Olver equation. Applied Mathematics and Computation. 2011;218:3962-3964.

25. EME Zayed, YA Amer. The modified simple equation method for solving nonlinear diffusive predator-prey system and Bogoyavlenskii equations. International Jornal of Physiacal Scienace. 2015;10(4):133-141.

26. EME Zayed, SA Hoda Ibrahim. Exact solutions of Kolmogorov-PetrovskiiPiskunov equation using the modified simple equation method. Acta Mathematicae Applicatae Sinica English Series. 2014;30(3):749-754.

27. NA Kudryashov. On one of methods for finding exact solutions of nonlinear differential equations, arXiv:1108.3288v1. 2011.

28. PN Ryabov, DI Sinelshchikov, MB Kochanov. Application of the Kudryashov method for finding exact solutions of the high order nonlinear evolution equations. Applied Mathematics and Computation. 2011;218:3965-3972.

29. EME Zayed, KAE Alurrfi. The homogeneous balance method and its applications for finding the exact solutions for nonlinear evolution equations. Italian Journal of Pure and Applied mathematics. 2014;33:307318.

30. WX Ma, Z Zhu. Solving the (3+1)-dimensional generalized KP and BKP equations by the multiple exp-function algorithms. Applied Mathematics and Computation. 2012;218:11871-11879.

31. WX Ma, T Huang, Y Zhang. A multiple exp-function method for nonlinear differential equations and its application. Phys Script. 2010;82:1-8.

32. WX Ma, JH Lee. A transformed rational function method and exact solutions to the (3+1)-dimensional Jimbo-Miwa equation. Chaos Solitons Fractls. 2009;42:1356-1363.

33. WX Ma, HY Wu, JS He. partial differential equations possessing Frobenius integrable decomposition technique. Physics Letters A. 2007;364:29-32.

34. YJ Yang, D Baleanu, XJ Yang. A Local fractional variational iteration method for Laplace equation within local fractional operators. Abstract and Applied Analysis. 2013.

35. AM Yang, XJ Yang, ZB Li. Local fractional series expansion method for solving wave and diffusion equations on cantor sets. Abstract and Applied Analysis. 2013.

36. EME Zayed, MAM Abdelaziz. The two variables $\left(\mathrm{G}^{\prime} / \mathrm{G}, 1 / \mathrm{G}\right)$ expansion method for solving the nonlinear KdV-mKdV equation. Mathematical Problems in Engineering. 2012.

37. EME Zayed, SA Hoda Ibrahim. The $\left(\mathrm{G}^{\prime} / \mathrm{G}, 1 / \mathrm{G}\right)$ expansion method and its applications for constructing the exact solutions of the nonlinear Zoomeron equation. World Journal of Modelling and Simulation. 2015;11(2):104110 . 
38. EME Zayed, KAE Alurrfi. The $\left(\mathrm{G}^{\prime} / \mathrm{G}, 1 / \mathrm{G}\right)$ expansion method and its applications to find the exact solutions of nonlinear PDEs for nanobiosciences. Mathematical Problems in Engineering. 2014.

39. EME Zayed, KAE Alurrfi. The $\left(\mathrm{G}^{\prime} / \mathrm{G}, 1 / \mathrm{G}\right)$ expansion method and its applications for solving two higher order nonlinear evolution equations. Mathematical Problems in Engineering. 2014.

40. EME Zayed, KAE Alurrfi. On solving two higher-order nonlinear PDEs describing the propagation of optical pulses in optic fibers using the $\left(\mathrm{G}^{\prime} / \mathrm{G}\right.$, 1/G) expansion method. Ricerche di Matematica. 2015;64(1):167-194.

41. SD Zhu. The generalized Riccati equation mapping method in nonlinear evolution equation: application to (2+1)-dimensional Boiti-leonPempinelle equation, Chaos, Solitons and Fractals. Science research publisher. 2008;37:1335-1342.

42. Z Li and X Zhang. new exact kink solutions and periodic form solutions for a generalized Zakharov-Kuznetsov equation with variable coefficients. Communications in Nonlinear Science and Numerical Simulation. 2010;15(11):3418-3422.
43. EME Zayed, AH Arnous. Many exact solutions for nonlinear dynamics of DNA model using the generalized Riccati equation mapping method. Scientific Research and Essays. 2013;8(8):340-346.

44. EME Zayed, YA Amer, RMA Shohib. The improved generalized Riccati equation mapping method and its application for solving a nonlinear partial differential equation (PDE) describing the dynamics of ionic currents along microtubules. Scientific Research and Essays. 2008;9(8):238-248.

45. HNaher, FA Abdullah, MA Akbar, A Yildirim. The extended generalized Riccati equation mapping method for the (1+1)-dimensional modified KdV equation. World Applied Sciences Journal. 2013;25(4):543-553.

46. EME Zayed, YA Amer. Exact solutions for the nonlinear KPP equation by using the Riccati equation method combined with the $\left(\mathrm{G}^{\prime} / \mathrm{G}\right)$ expansion method. Scientific Research and Essays. 2015;10(3):86-96. 